Marquette University

e-Publications@Marquette

Exercise Science Faculty Research and

Publications

Exercise Science, Department of

8-2018

\title{
Phase-III, Randomized Controlled Trial of the Behavioral Intervention for Increasing Physical Activity in Multiple Sclerosis: Project BIPAMS
}

\author{
Robert W. Motl \\ University of Alabama, Birmingham \\ Brian M. Sandroff \\ University of Alabama - Birmingham \\ Brooks C. Wingo \\ University of Alabama - Birmingham \\ Justin McCroskey \\ University of Alabama - Birmingham \\ Lara A. Pilutti \\ University of Ottawa
}

See next page for additional authors

Follow this and additional works at: https://epublications.marquette.edu/exsci_fac

\section{Recommended Citation}

Motl, Robert W.; Sandroff, Brian M.; Wingo, Brooks C.; McCroskey, Justin; Pilutti, Lara A.; Cutter, Gary R.; Bollaert, Rachel; and McAuley, Edward, "Phase-III, Randomized Controlled Trial of the Behavioral Intervention for Increasing Physical Activity in Multiple Sclerosis: Project BIPAMS" (2018). Exercise Science Faculty Research and Publications. 160.

https://epublications.marquette.edu/exsci_fac/160 


\section{Authors}

Robert W. Motl, Brian M. Sandroff, Brooks C. Wingo, Justin McCroskey, Lara A. Pilutti, Gary R. Cutter, Rachel Bollaert, and Edward McAuley 
Marquette University

e-Publications@Marquette

\section{Exercise Science Faculty Research and Publications/College of Health} Sciences

This paper is NOT THE PUBLISHED VERSION; but the author's final, peer-reviewed manuscript. The published version may be accessed by following the link in th citation below.

Contemporary Clinical Trials, Vol. 71 (August 2018): 154-161. DOI. This article is (C) Elsevier and permission has been granted for this version to appear in e-Publications@Marquette. Elsevier does not grant permission for this article to be further copied/distributed or hosted elsewhere without the express permission from Elsevier.

\section{Phase-III, Randomized Controlled Trial of the Behavioral Intervention for Increasing Physical Activity in Multiple Sclerosis: Project BIPAMS}

Robert W. Motl

Department of Physical Therapy, University of Alabama at Birmingham, AL

Brian M. Sandroff

Department of Physical Therapy, University of Alabama at Birmingham, AL

Brooks C. Wingo

Department of Occupational Therapy, University of Alabama at Birmingham, AL

Justin McCroskey

Department of Physical Therapy, University of Alabama at Birmingham, AL

Lara A. Pilutti

Interdisciplinary School of Health Sciences, University of Ottawa, Canada

Gary R. Cutter

Department of Biostatistics, University of Alabama at Birmingham, AL 


\section{Rachel E. Bollaert}

Department of Kinesiology and Community Health, University of Illinois at Urbana-Champaign, IL

\section{Edward McAuley}

Department of Kinesiology and Community Health, University of Illinois at Urbana-Champaign, IL

\section{Abstract}

\section{Background}

We propose a phase-III, randomized controlled trial (RCT) that examines the effectiveness of a behavioral intervention based on social cognitive theory (SCT) and delivered through the Internet using e-learning approaches for increasing physical activity and secondary outcomes (e.g., symptoms) in a large sample of people with multiple sclerosis (MS) residing throughout the United States.

\section{Methods/design}

The proposed phase-III trial will use a parallel group, RCT design that examines the effect of a 6-month behavioral intervention for increasing physical activity and secondarily improving mobility, cognition, symptoms, and quality of life $(\mathrm{QOL})$ in persons with MS. The primary outcome is accelerometer-measured moderate-tovigorous physical activity (MVPA). The secondary outcomes include self-report measures of physical activity, walking impairment, cognition, fatigue, depression, anxiety, pain, sleep quality, and QOL. The tertiary outcomes are mediator variables based on SCT. Participants $(N=280)$ will be randomized into behavioral intervention ( $n=140)$ or attention and social contact control $(n=140)$ conditions using computerized random numbers with concealed allocation. The conditions will be administered over 6 -months by persons who are uninvolved in screening, recruitment, random assignment, and outcome assessment. There will be a 6-month follow-up without intervention access/content. We will collect primary, secondary, and tertiary outcome data every 6 months over the 12-month period. Data analysis will involve intent-to-treat principles and latent growth modeling (LGM).

\section{Discussion}

The proposed research will provide evidence for the effectiveness of a novel, widely scalable approach for increasing lifestyle physical activity and improving secondary outcomes and QOL in persons with MS.

\section{Keywords}

Multiple sclerosis, Physical activity, Behavior change, Theory, E-learning

\section{Introduction}

Physical activity is lower among persons with multiple sclerosis (MS) than the general population [1]. This is problematic considering the exceedingly low rate of physical activity in the general population [2], evidence that physical activity levels decline over time in MS $[\underline{3}, \underline{4}]$, and observation that physical activity benefits might be greater in this population []․

The standard approach for promoting physical activity in MS has involved structured, supervised exercise training [6]. That approach has resulted in evidence of considerable benefits [ $\underline{5}$ ], but it has not altered the rate of physical inactivity within MS over the past 25 years [1]. Researchers have proposed moving away from structured exercise training, and focusing on behavioral interventions for changing lifestyle physical activity (i.e., accumulation of physical activity through planned or unplanned leisure, occupation, or household activities as part of everyday life) in MS $[\underline{7}, \underline{8}]$. Such behavioral interventions teach people the skills, techniques, and strategies for changing physical activity, typically based on a health behavior theory [ $\underline{7} \underline{8}]$. 
Researchers have developed, refined, and tested a behavioral intervention based on Social Cognitive Theory (SCT) [9] and have delivered this through an Internet website for changing physical activity and secondary symptomatic outcomes in MS [[10], [11], [12], [13]]. Although efficacious, the major weakness of that previous approach identified through formative evaluation $[\underline{10}, \underline{11}, \underline{13}]$ was the reliance upon content delivered through a text-based medium (i.e., participants read online text and passages for learning about principles of behavior change). That medium offered a passive, non-interactive, and non-immersive approach for learning the skills, techniques, and strategies necessary for changing physical activity. Such a weakness may be overcome through the adoption of e-learning software that permits the development of interactive video courses that seemingly provide an engaging user experience through pre-built and customizable interactions; slide layers and triggers; and a more immersive, engaging learner experience (see https://articulate.com/360/storyline). The elearning approach may increase the interest, processing, and accessibility of information by users for a greater impact of the behavioral intervention.

We recently completed a 6-month, phase-II, randomized controlled trial (RCT) that examined the efficacy of a newly developed Internet website that delivered a SCT-based behavioral intervention using e-learning approaches for increasing physical activity and improving symptoms, walking impairment, and neurological disability [14]. Participants with MS $(N=47)$ were randomly assigned into behavioral intervention $(n=23)$ or waitlist control $(n=24)$ conditions. Outcome assessments were administered before and after the 6-month study period. There were positive intervention effects on self-reported and objectively-measured moderate-tovigorous physical activity (MVPA), as well as on fatigue, depression and anxiety symptoms, walking mobility, and disability status. Such outcomes provide proof-of-principle evidence for a large, phase-III RCT testing the effectiveness of this approach for improving physical activity and secondary outcomes as well as examining mediators based on SCT (e.g., self-efficacy or goal setting).

The planned phase-III RCT will test the effectiveness of the behavioral intervention [14] for increasing physical activity and improving secondary outcomes among a large sample of people with MS residing throughout the United States. The primary objective examines the immediate and sustained effect of the behavioral intervention on objectively-measured MVPA; MVPA was deemed the primary end-point considering its importance for overall health as well as prevention of chronic disease and premature mortality. The secondary objective examines the immediate and sustained effect of the behavioral intervention on self-reported measures of physical activity, walking mobility, cognition, symptoms, disability status, and quality of life (QOL). The tertiary objective examines SCT variables as mediators of the behavioral intervention effect on change in physical activity.

\section{Methods}

\subsection{Study design and overview}

The proposed phase-III trial will use a parallel group, RCT design. The primary outcome is accelerometry as an objective measure of minutes/day of MVPA. The secondary outcomes include self-report measures of physical activity, walking mobility, cognition, fatigue, depression, anxiety, pain, sleep quality, and QOL. The tertiary outcomes are mediator variables based on SCT. Participants $(N=280)$ will be randomized into the behavioral intervention condition $(n=140)$ or an attention and social contact control condition $(n=140)$ using a random numbers sequence with concealed allocation. The conditions will be administered over six months by trained behavior coaches who will be uninvolved in screening, recruitment, random assignment, and outcome assessment. There will be a six-month follow-up period wherein participants will not access the study website nor engage in video chats with behavior coaches; this is important for determining if the behavioral intervention results in relatively permanent and stable levels of physical activity based on the logic of teaching the participants skills, strategies, and approaches for initiating and maintaining behavior change over time. We will 
collect primary, secondary, and tertiary outcome data every six months over the 12-month period(i.e., baseline, immediate follow-up, and 6-month follow-up). The data analyses will involve intent-to-treat principles and latent growth modeling (LGM). This study does not include a data safety monitoring board as it is a low risk, behavioral intervention with minimal side effects conducted in a population that is not identified as vulnerable and has been approved by an Institutional Review Board.

\subsection{Participants}

We will recruit a sample of 280 persons with MS from across the United States through postal and electronic advertisements delivered using the National MS Society (NMSS), NARCOMS, and iConquerMS. We further will distribute advertisements among the MS Centers identified through the NMSS website(https://www.nationalmssociety.org/Treating-MS/Find-an-MS-Care-Provider), and request that the materials are distributed among persons living with MS who visit the Centers for services. The advertisements will describe the study as comparing two different approaches delivered through the Internet for managing consequences of MS and improving health indicators. Those interested in participation will contact the study project coordinator either by e-mail or telephone; we will establish a toll-free telephone number considering the nationwide recruitment effort. This initial e-mail or telephone call will be followed-up by a phone call from the project coordinator who will describe the study and its procedures, answer all questions, and conduct a screening for inclusion criteria. The inclusion criteria involve (a) diagnosis of MS; (b) relapse free in the past 30 days; (c) Internet and email access; (d) willingness to complete the questionnaires, wear the accelerometer, and undergo randomization; (e) being non-active defined as not engaging in regular physical activity (30 min accumulated per day) on more than two days of the week during the previous six months (i.e., not meeting current physical activity guidelines for MS); (f) ability to ambulate with or without assistance (i.e., walking with or without a cane or walker, but not a wheelchair) as this intervention focuses on walking as a main agent of changing lifestyle physical activity behavior; and (g) age between 18 and 64 years. We will exclude all individuals with moderate or high risk for contraindications of possible injury or death when undertaking strenuous or maximal exercise using the Physical Activity Readiness Questionnaire (PAR-Q; [15]); this is a simple, self-reported screening tool for determining safety and possible risk for starting a physical activity program. During the initial phone contact with the project coordinator, participants will verbally respond to the 7 -items on the PAR-Q, and those individuals who report no more than one YES or affirmative on the seven items on the PAR-Q will be considered at low risk and included for participation. All other individuals will be considered at moderate or high risk for starting a physical activity program and excluded from participation, and further advised to seek medical guidance before becoming more physically active.

\subsection{Sample size}

We conducted a conventional power analysis to estimate the appropriate sample size for detecting a Condition ( 2 levels of between-subjects factor: Intervention vs. Control) $\times$ Time (3 levels of within-subjects factor: 0, 6, \& 12 months) interaction on the primary outcome of objectively-measured MVPA. The medium effect size (Cohen's $d=0.35$ ) for the power analysis was from our recent pilot RCT [14] of the same behavioral intervention compared with a waitlist control condition for increasing minutes/day of MVPA in persons with MS. The power analysis included assumptions of reliability for the within-subjects factor of ICC $=0.50$, two-tailed $\alpha=0.05$, and $\beta=0.05$ (i.e., $95 \%$ power). The power analysis indicated that the minimal total sample size for testing the Time $\times$ Condition interaction on physical activity should be nearly 240 participants. This would support our goal of recruiting 280 participants as yielding adequate power, based on retention of $85 \%$ of the participants. We have successfully retained over $90 \%$ of participants over six months in our pilot trial of the behavioral intervention [14]. This sample will be particularly appropriate and necessary considering our data analyses will involve LGM. 


\subsection{Measures}

\subsubsection{Primary outcome}

The primary study outcome is objectively-measured minutes/day of MVPA over a seven-day period (i.e., previous week); this will be measured by ActiGraph model GT3X+ accelerometers (Actigraph Corporation, FL). The accelerometer will be placed in a pouch on an elastic belt that is worn around the waist over the nondominant hip during the waking hours of a seven-day period. The data from the accelerometer will be downloaded, processed using the low frequency extension (i.e., filter for increasing the devices sensitivity for lower intensity physical activity and resulting in comparability with previous accelerometer models) into oneminute epochs using Actilife (Actigraph Corporation, FL) software, and then scored for wear time and minutes/day of MVPA based on activity count cut-points for MS [16]; the filter yields data from the GT3X+ that are comparable with the model 7164 wherein the MVPA cut-points were derived. Only data from valid days (wear time $\geq 600 \mathrm{~min}$ ) will be included in the analyses. We will compare the groups for number of valid days and we will average the data over two or more valid days for the outcome of minutes/day of MVPA during the previous week, as this provides a reliable estimate of physical activity behavior [17]. The accelerometer data will permit the generation of other measures such as steps per day and time spent in light physical activity and sedentary behavior as exploratory end-points.

\subsubsection{Secondary outcomes}

Physical activity will be further measured using the Godin Leisure-Time Exercise Questionnaire (GLTEQ; [18]) and the abbreviated International Physical Activity Questionnaire (IPAQ; [19]). The inclusion of two additional selfreports permits the cross-validation of behavior change and capturing of possible unique changes in physical activity. The GLTEQ includes three items that measure the frequency of engagement in mild, moderate, or strenuous physical activity for at least 15 min during one's leisure-time in the previous week. We will compute the health contribution score (HCS) for the GLTEQ by multiplying the frequency of strenuous and moderate activity by nine and five metabolic equivalents of task (METS), respectively, and then summing the weighted scores [20]. The HCS is a valid measure of MVPA in persons with MS [21]. The abbreviated IPAQ was designed for population surveillance of physical activity among adults and contains 6 items that measure the frequency and duration of vigorous-intensity activities, moderate-intensity activities, and walking during a seven-day period. We will not include the duration component in this study based on previous research that identified problems with accurate recall of physical activity duration in persons with MS [22]. The respective frequency values for vigorous, moderate, and walking activities will be multiplied by eight, four, and 3.3 metabolic equivalents and then summed to form a continuous measure of physical activity.

Walking mobility will be measured by the Multiple Sclerosis Walking Scale-12 (MSWS-12; [23]). The MSWS-12 provides a 12-item, patient-reported outcome of walking impairment over the past two weeks [23]. Cognitive function will be assessed using the Perceived Deficits Questionnaire (PDQ; [24]) and MS Neuropsychological Questionnaire (MSNQ; [25]). The PDQ provides a self-report of cognitive dysfunction in MS and contains 20 items that measure perceived problems with memory, attention, and concentration over the past four weeks. The MSNQ is a self-administered questionnaire that contains 15 items and measures the frequency of experiencing problems with attention, processing speed, memory, and other cognitive functions during the past three months. Symptoms of fatigue, depression, anxiety, pain, and sleep quality will be measured by the Fatigue Severity Scale (FSS; [26]), Modified Fatigue Impact Scale (MFIS; [27]), Hospital Anxiety and Depression Scale (HADS; [28]), short-form McGill Pain Questionnaire (SF-MPQ; [29]), and Pittsburgh Sleep Quality Index (PSQI; [30]), respectively. The FSS is a nine item unidimensional measure of fatigue and its disabling consequences over the past week in medical populations including MS. The MFIS is a 21-item measure of physical, cognitive, and psychosocial impact of fatigue on daily life over the past four weeks. The HADS contains 14 items that measure the frequency of anxiety and depressive symptoms over the past week. The SF-MPQ has 
a 15-item adjective checklist that captures sensory and affective dimensions of pain experienced over the past week. The PSQI is a 19-item, self-rated questionnaire that yields a global score of sleep quality over the past month. QOL will be measured by the 36-item Short Form Health Survey (SF-36; [31]) and Multiple Sclerosis Impact Scale-29 (MSIS-[29]; [32]). The SF-36 is a generic, 36-item measure of 8 subdomains and physical and mental components of health-related QOL over the past four weeks. The MSIS-29 is a 29-item measure of mental and physical domains of QOL over the past four weeks developed for people with MS.

The Patient Determined Disease Steps (PDDS; [33]) scale will characterize the overall level of disability of the sample and capture possible change in disability. The PDDS is a single, nine-point item for reporting neurological impairment on a scale of 0 (Normal) through 8 (Bedridden) with a midpoint of score of 4 (Early cane).

\subsubsection{Tertiary outcomes}

The SCT mediator variables will include self-efficacy, outcome-expectations, goal setting/planning, and facilitators/impediments. Self-efficacy will be measured by the six-item Exercise Self-Efficacy scale [34] and the 13-item Barriers Specific Self-Efficacy scale [35]. Outcome expectations for exercise will be measured by the 15item Multidimensional Outcomes Expectations for Exercise Scale [36]. Goal-setting and planning will be measured with the 10-item Exercise Goal-Setting scale and the 10-item Exercise Planning scale [37]. We will measure functional limitations as an impediment using the 15-item Functional Limitations component of the abbreviated Late Life-Function and Disability Inventory [38]. We will measure social support as a facilitator using the six-item Social Provisions Scale [39].

\subsubsection{Engagement}

We will track engagement with the BIPAMS and WellMS websites by monitoring logins. We will further track engagement based on attendance during the scheduled video chat sessions for both conditions.

\subsection{Procedures}

The study procedures mimic our previous research [14] and have been reviewed and approved by a university institutional review board; the trial is registered at ClinicalTrials.gov (NCT03490240). Interested participants will contact the project coordinator who will describe the study (i.e., comparing two approaches delivered through the Internet for managing consequences of MS and improving health indicators) and what it entails, and then conduct a screening for inclusion criteria over the phone. The project coordinator will then distribute the informed consent document among participants who satisfy inclusion criteria through email and postal mail. This will be followed by a phone call to ensure that the participants received the document, understand the study and its procedures, and sign the informed consent. Participants will complete enrollment by returning a signed copy of this document. Once enrolled, the project coordinator will contact participants and schedule baseline data collection.

The project coordinator will send the participant a packet of questionnaires and an accelerometer via certified postal mail; the packet of questionnaires will take an estimated 60-90 min for completion. This packet will include instructions for reminding the participants about the importance of wearing the accelerometer as instructed every day during the seven-day period, and provide a pre-stamped and pre-addressed envelope for return postal service. The participants will wear the accelerometer for a seven-day period. The project coordinator will send brief e-mails and make short phone calls for reminding participants about wearing the accelerometer every day in the middle of the seven-day period. This will be followed by a phone call to make sure the participants wore the accelerometer daily during the seven-day period and returned it through the US postal service. Once the baseline assessment is completed, participants will be randomly assigned into either the intervention (Behavioral Intervention for Physical Activity in Multiple Sclerosis; BIPAMS) or control (Wellness for MS; WellMS) condition using a random numbers sequence with concealed allocation (i.e., opaque sealed envelopes) by a member of the research team uninvolved in delivering the intervention. The 
intervention/control conditions will be delivered in four waves of $\sim 70$ participants per wave, and the conditions will be delivered across a 6 -month period. Participants will be asked to contact the project coordinator via the dedicated toll-free number or e-mail in the occurrence of an adverse event or any other problem; this information will further be collected during video chats with behavioral coaches. The participants will complete the same measurement procedures immediately (i.e., immediate follow-up) and six-months (i.e., six-month follow-up) after the intervention/control conditions. There will be no website access or chats during the sixmonth follow-up period for examining sustainability. Participants will receive $\$ 50$ USD remuneration for completing the measures per measurement period, including the baseline measurement, for a total of \$150 USD. We will further collect formative feedback using a survey with standard response options and open-ended items for identifying opportunities for intervention improvement moving forward.

\subsection{Behavioral intervention - BIPAMS}

As described previously [14], the behavioral intervention consists of two primary components, namely a dedicated Internet website and one-on-one video chats with a behavioral coach (see Table 1). The Internet website was programed in Drupal ${ }^{\mathrm{TM}} 8.0$ for accessibility, and underwent modification from previous versions based on the research team's experiences with MS and focus group feedback, as well as formative evaluation from pilot testing for efficacy in multiple published RCTs [[10], [11], [12], [13]]. The most extensive modification involved the transition from a primarily text-based presentation of material in previous versions of the behavioral intervention [[10], [11], [12], [13]] into interactive video courses designed based on e-learning principles. We further included a physical activity "Tracker" feature embedded directly within the website itself rather than as an external tool. This allowed participants to directly enter weekly step counts and graphically monitor activity levels within the Internet website. The Internet website is password protected and hosted on a research-based HIPAA-compliant server.

Table 1. Description of intervention components and elements for the BIPAMS and WellMS interventions.

\begin{tabular}{|l|l|l|l|}
\hline $\begin{array}{l}\text { Intervention } \\
\text { Components }\end{array}$ & Elements within Component & BIPAMS & WellMS \\
\hline Internet Website & Target & Physical Activity & General Wellness \\
\hline & $\begin{array}{l}\text { Source of intervention } \\
\text { content }\end{array}$ & $\begin{array}{l}\text { Social Cognitive } \\
\text { Theory }\end{array}$ & $\begin{array}{l}\text { National Multiple Sclerosis } \\
\text { Society }\end{array}$ \\
\hline & Interactive video courses (\#) & 10 & 10 \\
\hline & Resource section & Yes & Yes \\
\hline & Learn more section & Yes & Yes \\
\hline & Tracker & Yes & No \\
\hline & Forum & Yes & Yes \\
\hline & Patient voices (\#) & 24 & 10 \\
\hline & Weekly email announcements & Yes & Yes \\
\hline & Weekly updates \\
announcements & Yes & Yes \\
\hline & Tips of the week & Yes & Yes \\
\hline One-on-One Video & News and events section & Yes & Yes \\
\hline Chats & Occurrence (\#) & 13 & 9 \\
\hline & Semi-scripted guide & Yes & Yes \\
\hline & Adverse event reporting & Yes & Yes \\
\hline Other & Pedometer & Yes & No \\
\hline & Goal-setting & Yes & Yes \\
\hline & Log books/self-monitoring & Yes & \\
\hline & & & Yes \\
\hline
\end{tabular}


Note. BIPAMS, Behavioral Intervention for Physical Activity in Multiple Sclerosis. WellMS, Wellness for Multiple Sclerosis.

\subsubsection{Internet website}

The Internet website represents a medium for disseminating information on the skills, techniques, resources, and strategies for becoming and staying physically active with MS. The primary content of the Internet website is based on SCT and represents the transformation of an effective and empirically validated, face-to-face intervention approach that increased adherence with supervised exercise training [40] in persons with MS [41]. The content is delivered through interactive video courses based on e-learning principles. The interactive video courses were developed using Storyline in Articulate 360 (Articulate,New York,NY) based on operationalizing principle elements of SCT including self-efficacy, outcome expectations, impediments, and goal-setting. The interactive video courses follow a similar template of Introduction and Overview, Primary Content, and Take Home Message and were developed for an eighth grade reading level and with steady presentation of animations. The interactive video courses received formative evaluation from representative persons with MS and are organized into four successive "Modules" for the behavioral intervention, namely Getting Started; Planning for Success; Beating the Odds; and Sticking with It. The Getting Started module is delivered first and includes interactive video courses on the benefits of physical activity for persons with MS, instructions for becoming physically active, completing daily activity logs, and self-monitoring of behavior via a Yamax SW200 pedometer and an embedded website feature called Tracker for reporting weekly physical activity. The Planning for Success module is delivered second and includes interactive video courses on goal setting and feedback again via a Yamax SW-200 pedometer and Tracker, developing realistic outcome expectations, and increasing self-efficacy. The Beating the Odds module is delivered third and includes interactive video courses on identifying barriers to physical activity and strategies of overcoming barriers as well as developing social support. The Sticking with It module is delivered fourth and includes interactive video courses on maintaining an active lifestyle and prevention of relapse into a sedentary lifestyle. The interactive video courses include embedded, supplementary options of "Learn More" and "Resources." The "Learn More" option includes passive videos on content that are aligned with the course, and the "Resources" option includes research articles that document the scientific evidence for statements within the courses; manuals on stretching, aerobic, and resistance modes of physical activity; and worksheets and questionnaires for further developing the personal relevance of the stories. The interactive video courses along with embedded options become "accessible" in a titrated fashion of seven times during the first 2 months, four times during the second 2 months, and twice during the final 2 months of the intervention.

The Tracker feature of the website is designed for self-monitoring and goal-setting within the website itself, and the data are directly accessible by the behavioral coaches for discussions within the one-on-one video chats. Tracker includes four elements of Ready (directly entering baseline week of step counts), Set (setting a step count goal for the program), Go (directly entering step counts weekly during the program), and See (graphical display of step counts for monitoring progress over the program compared with the goal). This is further supplemented with direct, on-screen feedback regarding progress with the behavioral goal on a weekly basis. We again received formative evaluation from representative persons with MS for refinement of the new Tracker feature prior to implementation.

The website further includes "Voices" or audio files of individual persons with MS discussing physical activity that are overlaid with loops of photographs of the narrator. The "Voices" change weekly and are included for the purpose of engaging people with MS in social modeling as a source of self-efficacy information based on SCT [9]. The Internet website further includes an ongoing participant "Forum" for discussions of physical activity behavior change among participants as another form of vicarious experience; the forum is monitored, but not moderated, by the study team. The website is supported by automated e-mail announcements about updates and tips of the week and includes information on News and Events that further supplement the website content. 


\subsubsection{One-on-one video chats}

The BIPAMS intervention further includes one-on-one video chats with participants. The video chats are delivered by behavioral coaches who are doctoral-level graduate students and post-doctoral fellows who have undertaken extensive coursework and training in behavior change theory and training on the application of SCT for MS. The one-on-one video chats support adherence to the intervention, discussion and elaboration of website material, supportive accountability, and reporting of adverse events/injuries; these further provide social persuasion for promoting behavior change. The video chats are modeled based on TeleCoach [41] for maximizing adherence with Internet interventions, provide an ongoing interaction between the behavioral coach and the participant, and are conducted face-to-face through Skype ${ }^{\mathrm{TM}}$. The video chats are semi-scripted and based on principles of supportive accountability. The video chats consist of an ongoing review of goalsetting and progress toward goal attainment through Tracker as well as discussion of strategies and facilitators of behavioral change based on SCT and current website content. The chats occur seven times during the first 2 months, four times during the second 2 months, and twice during the final 2 months of the intervention; this aligns with the accessibility of new interactive video courses. The video chats further allow for inquiry and reporting on adverse and serious adverse events.

\subsection{Control condition - WellMS}

The previous pilot trials of behavioral interventions for increasing physical activity in MS have included a passive, waitlist control $[\underline{10}, 13]$. The current study improves upon those previous RCTs by providing an Internet website and one-on-one video chats with behavioral coaches that discuss materials about self-managing MS symtpoms and health indicators through methods other than physical activity (i.e., active control condition; see Table 1 for comparison of components with the BIPAMS condition). The materials are transformations of brochures provided by the NMSS, including Gait or Walking Problems: The Basic Facts; MS and Your Emotions; Pain: The Basic Facts; Solving Cognitive Problems; Taming Stress in MS; Food for Thought: MS and Nutrition; and Vitamins, Minerals, and Herbs: An Introduction. The delivery of the Internet materials and chat sessions will occur on the same time schedule and frequency as the intervention condition, and will have a comparable time commitment. The website further includes "Voices" of individual persons with MS discussing wellness strategies that are overlaid with loops of photographs of the narrator. As is the case for the behavioral intervention condition, the Internet website includes an ongoing and seeded participant "Forum" for discussions of wellness among participants. The website is supported by automated e-mail announcements about updates and tips of the week and includes information on News and Events that further supplement the website content. The Internet website is password protected and hosted on a research-based HIPAA-compliant server. The control condition will account for attention and social contact as well as other possible biases such as initial reactivity and time spent on the website and video chats. The control condition is consistent with the definition of an NIH-defined phase-III RCT [42]. Importantly, the participants in the control condition will not be offered the behavioral intervention for increasing physical activity after completion of the study procedures. The control condition further will not involve the application of "Tracker" nor the provision of pedometers for self-monitoring physical activity behavior.

\subsection{Data analysis}

The data will be coded and double entered on a computer by trained laboratory personnel. The data will then be examined for errors (e.g., out of range values) using frequency analyses in IBM SPSS Statistics 24 . The data analysis will proceed with a final, error-free data set. The first step in the data analysis will be an inspection for outliers and normality violations. The screening for outlying values is necessary for identifying data points that might distort the data analysis and interpretation. The screening for normality violations is important in light of the assumptions of the subsequent data analysis. Screening for outliers and normality violations will be undertaken using inspection of frequency distributions and plots (i.e., graphical methods such as histograms and 
box plots), estimation of z-scores, estimation of skewness and kurtosis, and test for normality (i.e., analytic methods). If necessary, we will transform the data points (i.e., downweighting or Winsorizing) or distributions (e.g., square root, logarithm, inverse, etc.) for minimizing problems with outliers or normality.

We will complete basic descriptive statistics and information necessary for completing the CONSORT diagram when starting the data analysis process. We will then undertake comparisons of the two conditions to identify any potential imbalances that may be considered for adjustment in subsequent analyses.

\subsubsection{General analytic framework}

The hypotheses will be addressed by analyzing the data with structural equation modeling (SEM) and a LGM framework. LGM is a powerful approach within SEM for simultaneously studying the pattern, predictors, and consequences of longitudinal change processes [[43], [44], [45], [46]]. LGM has a number of advantages over other more commonly adopted approaches used to study change among continuous variables (e.g.,

ANOVA, MANOVA, lagged regression, use of change scores) including the ability to (a) model change at the individual as well as the group-level of analysis; (b) model individual differences in change trajectories (initial status and slope factors); (c) model various functional forms of change (e.g., linear and quadratic); (d) model change in several focal variables concomitantly; and (e) directly model important predictors and outcomes of longitudinal change [[43], [44], [45], [46]]. The LGM analyses will be performed using the fullinformation maximum likelihood (FIML) estimator as fit function and the Mplus software package [47]. The FIML estimator allows for the estimation of models in the presence of missing data (i.e., intent-to-treat), and has yielded relatively unbiased parameter estimates, standard errors, and fit indices under conditions of $\leq 25 \%$ missing data ([표]). Model-data fit will be evaluated using accepted standards. Good model-data fit will be established based on a root mean square error of approximation of $\leq 0.06$, non-normed and comparative fit indices of $\geq 0.95$, and standardized root mean residual $\leq 0.08$ [49].

The hypotheses for the primary and secondary endpoints (i.e., behavioral intervention will increase objectivelymeasured MVPA and, secondarily, improve self-reported physical activity, walking mobility, cognition, symptoms, and QOL in persons with MS) will be tested using a two-step approach in LGM. The first step will involve single-sample LGM for estimating the trajectory or pattern of change in MVPA and self-reported physical activity, walking mobility, cognition, symptoms, and QOL across the measurement occasions in the intervention and control groups separately. The second step will involve two-sample LGM for testing differences in the trajectory or pattern of change in physical activity, for example, between the intervention and control groups, based on the difference in slope coefficients using chi-square tests. This approach will afford estimates of both the within-subjects and between-subjects pattern of change. We will conduct exploratory analyses of clinical (i.e., disease duration and MS type), demographic (e.g., sex and age), and pharmacologic (e.g., disease-modifying medications and symptomatic medications) variables that might influence the pattern of growth on the primary and secondary outcome variables (i.e., moderators of intervention heterogeneity); this will identify for whom the BIPAMS intervention works in promoting behavior change and secondary benefits.

The hypothesis for tertiary endpoints (i.e., SCT variables as mediators of the behavioral intervention effect on change in physical activity) will be tested using LGM with parallel growth processes [47]. The LGM with parallel growth processes includes the standard LGM for establishing the pattern or trajectory of change in MVPA and self-reported physical activity and the mediator variables separately. The LGM with parallel growth processes further includes path coefficients $(\beta s)$ between the initial status latent variables and between the change or slope latent variables. Within this parallel growth process LGM, change in self-efficacy will predict change in physical activity directly and indirectly by way of outcome expectations, impediments, and goal setting, as proposed by SCT [[7], [8], [9]] and supported in our cross-sectional [50] and longitudinal [51] data. We will test the direct and indirect pathways using the INDIRECT option available in the Mplus software package [47]. This 
approach will again involve two-sample analysis for testing the hypothesized model with parallel growth processes in the intervention and control conditions separately and then directly testing the equivalence or difference in the proposed relationships between the two conditions using chi-square tests.

\section{Discussion}

The proposed behavioral intervention focuses on changing one of the more important lifestyle behaviors, namely physical activity, and examining its immediate and sustained influence on self-reported walking mobility, cognition, symptoms, and QOL in persons with MS. To date, researchers have reported positive effects of faceto-face or supervised delivery of exercise training interventions on mobility, symptoms, and QOL in persons with $\operatorname{MS}[\underline{5}, \underline{6}]$, but this population continues to engage in minimal amounts of physical activity compared with the general population of adults [1] despite those benefits. This underscores a critical opportunity to improve mobility, cognition, symptoms, and QOL in persons with MS by developing interventions for increasing lifestyle physical activity behavior. Importantly, the traditional approach of face-to-face delivery has limited reach, efficiency, and accessibility for the target population and is impractical for increasing lifestyle physical activity, particularly in large-scale dissemination projects among persons with MS who face considerable barriers associated with accessing an exercise facility []]. To that end, the Internet represents an ideal medium for delivering a behavioral intervention that increases physical activity, particularly based on the Internet use [52] and interests [53] of persons with MS. The results of the proposed phase-III RCT would provide an empirical basis for the effectiveness of a behavioral intervention based on SCT and delivered via the Internet for increasing physical activity with secondary effects on walking mobility, cognition, symptoms, and QOL in persons with MS.

This study is adopting a critical focus on promoting and sustaining lifestyle physical activity behavior, rather than structured exercise training, and examining its influence on walking mobility, cognition, symptoms, and QOL in persons with MS. The existing research has largely focused on the effects of structured, supervised exercise training for influencing mobility, symptoms, and QOL in MS $[\underline{5}, \underline{6}]$. We argue that such programs are exceedingly valuable, but have little relevance for understanding the secondary benefits of change in free-living physical activity. Physical activity that occurs in the context of everyday life is much more accessible for persons with MS, and represents a novel focus on managing mobility impairment and worsening of symptoms and QOL in this population. The focus on lifestyle physical activity further reflects a paradigm shift that has evolved over the past decade in the general population [54] and that is now being considered in MS [7, 8]. Our previous data support associations among free-living physical activity, walking mobility, cognition, symptoms, and QOL [8], but we are unaware of large-scale interventions designed for increasing and sustaining this behavior and examining its influence on meaningful outcomes in persons with MS. Indeed, face-to-face delivery is effective, but suffers from a major problem with dissemination, particularly among those without access to exercise facilities and trained exercise leaders who can facilitate the delivery. Using the Internet for delivery of the behavioral interventions, rather than face-to-face interactions, presents an alternative approach for increasing free-living physical activity among those with MS and further reflects a cost-effective method of increasing physical activity in this population that can be broadly disseminated with greater potential reach, efficiency, accessibility, and sustainability.

Another relevant feature of the proposed research is the inclusion of an active and credible control condition. Indeed, previous pilot trials of behavioral interventions for increasing physical activity in MS have included a passive, waitlist control $[\underline{10}, \underline{13}]$. The current study improves upon those previous RCTs by providing a control condition that involves an Internet website and one-on-one video chats with behavioral coaches that discuss materials about self-managing MS symptoms and health indicators through methods other than physical activity. The materials are transformations of brochures provided by the NMSS, including Gait or Walking Problems: The Basic Facts; MS and Your Emotions; Pain: The Basic Facts; Solving Cognitive Problems; Taming 
Stress in MS; Food for Thought: MS and Nutrition; and Vitamins, Minerals, and Herbs: An Introduction. This condition will not likely influence physical activity behavior or mediator variables, as these are physical activityspecific; however, this condition could improve secondary outcomes such as fatigue and depression, but probably not as strongly as the physical activity intervention.

One final relevant feature of the proposed research is the focus on putative mediators of the behavioral intervention for increasing physical activity among persons with MS. Mediators are intermediate variables in the causal sequence between the independent and dependent variables, and essentially explain why interventions are effective for changing outcome variables, in this case physical activity behavior. Our previous research indicates that self-efficacy has been directly and indirectly associated with physical activity in persons with MS, and the indirect effects have operated through outcome-expectations, impediments, and goal setting; this set of relationships is consistent with SCT [9]. We further note that the current behavioral intervention for increasing physical activity targets key constructs of SCT. To that end, the results of the mediator analysis will provide novel information about mechanisms of change in physical activity among persons with MS. The information on mediators will inform and refine our subsequent research by identifying the most relevant variables that should be the targets of a behavioral intervention. Such information might provide confirmation of the primary causative agents from Bandura's SCT [9] for designing RCTs, and will ultimately assist in clarifying the relevant aspects of SCT for promoting physical activity behavior change in persons with MS.

There are several important limitations of the proposed phase-III RCT. One limitation is that we are measuring the outcomes using paper-and-pencil surveys rather than online data collection. We decided on paper-andpencil surveys, as the measurement properties of surveys can change when delivering them through another medium such as online, and this could serve as a threat toward interpreting the outcomes of the study. We further were already delivering the accelerometer through the mail, and have successfully provided the paperand-pencil surveys along with the accelerometer in our previous research. Another limitation is that we are not collecting data on health economics, but we will work with the NMSS, the funder of this research, on the roll-out of the BIPAMS intervention, if the results are sufficiently positive. The intervention is currently only available in English and therefore is not transferable into non-English speaking countries. We are only collecting follow-up data for 6-month post-intervention cessation, and this might be considered a medium-term rather than longterm outcome.

Collectively, the proposed phase-III RCT will test the effectiveness of the aforementioned behavioral intervention [14] for increasing physical activity and improving secondary outcomes among a large sample of people with MS residing throughout the United States. The primary objective examines the immediate and sustained effect of the behavioral intervention on objectively-measured MVPA. The secondary objective examines the immediate and sustained effect of the behavioral intervention on self-reported measures of physical activity, walking mobility, cognition, symptoms, disability status, and QOL. The tertiary objective examines SCT mediators for the effect of the behavioral intervention on change in physical activity. This research, once completed, will further permit examinations of response heterogeneity in physical activity and secondary end-points, and exploration of moderating factors that might explain the variation in intervention effectiveness. Such research will present a significant and substantial advancement in the study of physical activity behavior change for improving the lives of persons with MS.

\section{Acknowledgement}

This study was funded by a grant from the National Multiple Sclerosis Society (RG 5144A6/1). 


\section{References}

[1] D. Kinnett-Hopkins, B. Adamson, K. Rougeau, R.W. Motl People with MS are less physically active than healthy controls but as active as those with other chronic diseases: an updated meta-analysis Mult. Scler. Relat. Disord., 13 (2017), pp. 38-43

[2] Centers for Disease Control and Prevention Prevalence of no leisure-time physical activity - $\mathbf{3 5}$ states and District of Columbia, 1988-2002 MMWR, 53 (2004), pp. 82-86

[3] R.W. Motl, E. McAuley, B.M. Sandroff Longitudinal change in physical activity and its correlates in relapsingremitting multiple sclerosis Phys. Ther., 93 (2013), pp. 1037-1048

[4] R.E. Klaren, J.E. Sasaki, E. McAuley, R.W. Motl Patterns and predictors of change in moderate-to-vigorous physical activity over time in multiple sclerosis J. Phys. Act. Health, 14 (2017), pp. 183-188

[5] B. Lai, H.J. Young, C.S. Bickel, R.W. Motl, J.H. Rimmer Current trends in exercise intervention research, technology, and behavioral change strategies for people with disability: a scoping review Am. J. Phys. Med. Rehabil., 96 (2017), pp. 748-761

[6] R.W. Motl, B.M. Sandroff, G. Kwakkel, U. Dalgas, A. Feinstein, C. Heesen, P. Feys, A.J. Thompson Exercise in patients with multiple sclerosis Lancet Neurol., 16 (2017), pp. 848-856

[7] T. Ellis, R.W. Motl Physical activity behavior change in persons with neurological disorders: overview and examples from Parkinson disease and multiple sclerosis J. Neurol. Phys. Ther., 37 (2013), pp. 85-90

[8] R.W. Motl Lifestyle physical activity in persons with multiple sclerosis: the new kid on the MS block Mult. Scler., 20 (2014), pp. 1025-1029

[9] A. Bandura Health promotion by social cognitive means Health Educ. Behav., 31 (2004), pp. 143-164

[10] R.W. Motl, D. Dlugonski, T.R. Wójcicki, E. McAuley, D. Mohr Internet intervention for increasing physical activity in persons with multiple sclerosis Mult. Scler., 17 (2011), pp. 116-128

[11] D. Dlugonski, R.W. Motl, E. McAuley Increasing physical activity in multiple sclerosis: replicating internet intervention effects using objective and self-report outcomes J. Rehabil. Res. Dev., 48 (2011), pp. 11291136

[12] D. Dlugonski, R.W. Motl, D.C. Mohr, B.M. Sandroff Internet-delivered behavioral intervention to increase physical activity in persons with multiple sclerosis: sustainability and secondary outcomes Psychol. Health Med., 17 (2012), pp. 636-651

[13] L.A. Pilutti, D. Dlugonski, B.M. Sandroff, R. Klaren, R.W. Motl Randomized controlled trial of a behavioral intervention targeting symptoms and physical activity in mutliple sclerosis Mult. Scler., 20 (2014), pp. 594-601

[14] R.W. Motl, E.A. Hubbard, R.E. Bollaert, B.C. Adamson, D. KinnettHopkins, J.M. Balto, S.K. Sommer, L.A. Pilutti, E. McAuley Randomized controlled trial of an e-learning designed behavioral intervention for increasing physical activity behavior in multiple sclerosis Mult. Scler. J. Exp. Transl. Clin., 3 (2017) (2055217317734886)

[15] S. Thomas, J. Reading, R.J. Shephard Revision of the physical activity readiness questionnaire Can. J. Sport. Sci., 17 (1992), pp. 338-345

[16] B.M. Sandroff, Y. Suh, R.W. Motl Accelerometer output and its association with energy expenditure in persons with multiple sclerosis J. Rehabil. Res. Dev., 49 (2012), pp. 467-475

[17] R.E. Klaren, E.A. Hubbard, W. Zhu, R.W. Motl Reliability of accelerometer scores for measuring sedentary and physical activity behaviors in persons with multiple sclerosis Adapt. Phys. Act. Q., 33 (2016), pp. 195204

[18] G. Godin, R.J. Shephard A simple method to assess exercise behaviour in the community Can. J. Appl. Sport. Sci., 10 (1985), pp. 141-146

[19] C.L. Craig, A.L. Marchall, M. Sjostrom, A.E. Bauman, M.L. Booth, B.E. Ainsworth, M. Pratt, U. Eklund, A. Yng 
ve, J.F. Sallis, P. Oja International physical activity questionnaire: 12-country reliability and validity Med.

Sci. Sports Exerc., 35 (2003), pp. 1381-1395

[20] G. Godin The Godin-Shephard leisure-time physical activity questionnaire Health Fit. J. Can., 4 (2011), pp. $18-22$

[21] R.W. Motl, B.M. Sandroff, R.E. Klaren Validation of the health composite score from the Godin leisure-time exercise questionnaire and its classification coding system using accelerometry in multiple sclerosis Rehabil. Psychol., 63 (2018), pp. 77-82

[22] J.L. Gosney, J.A. Scott, E.M. Snook, R.W. Motl Physical activity and multiple sclerosis: validity of self-report and objective measures Fam. Commun. Health, 3 (2007), pp. 144-150

[23] J.C. Hobart, A. Riazi, D.L. Lamping, R. Fitzpatrick, A.J. Thompson Measuring the impact of MS on walking ability: the 12-item MS walking scale (MSWS-12) Neurology, 60 (2003), pp. 31-36

[24] J.J.L. Sullivan, K. Edgley, E. Dehoux A survey of multiple sclerosis, part 1: perceived cognitive problems and compensatory strategy use Can. J. Rehabil., 4 (1990), pp. 99-105

[25] R.H. Benedict, F. Munschauer, R. Linn, C. Miller, E. Murphy, F. Foley, L. Jacobs Screening ofr multiple sclerosis cognitive impairment using a self-administered 15-item questionnaire Mult. Scler., 9 (2003), pp. 95-101

[26] L.B. Krupp, N.G. Larocca, J. Muir-Nash, A.D. Steinberg The fatigue severity scale. Application to patients with multiple sclerosis and systemic lupus erythematosus Arch. Neurol., 46 (1989), pp. 1121-1123

[27] J.D. Fisk, P.G. Ritvo, L. Ross Measuring the functional impact of fatigue: initial validation of the fatigue impact scale Clin. Infect. Dis., 18 (1994), pp. S79-S83

[28] A.S. Zigmond, R.P. Snaith The hospital anxiety and depression scale Acta Psychiatr. Scand., 67 (1983), pp. 361-370

[29] R. Melzack The short-form McGill pain questionnaire Pain, 30 (1987), pp. 191-197

[30] D.J. Buysse, C.F. Reynolds, T.H. Monk The Pittsburgh sleep quality index: a new instrument for psychiatric practice and research Psychiatry Res., 28 (1989), pp. 193-213

[31] J. Ware, C. Sherbourne The MOS 36 item short-form health survey (SF-36) Med. Care, 36 (1992), pp. $473-$ 483

[32] J. Hobart, D. Lamping, R. Fitzpatrick The multiple sclerosis impact scale (MSIS-29): a new patient-based outcome measure Brain, 124 (2001), pp. 962-973

[33] O. Hadjimichael, R.B. Kerns, M.A. Rizzo, G. Cutter, T. Vollmer Persistent pain and uncomfortable sensations in persons with multiple sclerosis Pain, 127 (2007), pp. 35-41

[34] E. McAuley The role of efficacy cognitions in the prediction of exercise behavior in middle-aged adults J. Behav. Med., 15 (1992), pp. 65-88

[35] E. McAuley Self-efficacy and the maintenance of exercise participation in older adults J. Behav. Med., 16 (1993), pp. 103-113

[36] E. McAuley, R.W. Motl, S.M. White, T.R. Wójcicki Validation of the multidimensional outcome expectations for exercise scale in individuals with multiple sclerosis Arch. Phys. Med. Rehabil., 91 (2010), pp. 100-105

[37] L.S. Rovniak, E.S. Anderson, R.A. Winett, R.S. Stephens Social cognitive determinants of physical activity in young adults: a prospective structural equation analysis Ann. Behav. Med., 24 (2002), pp. 149-156

[38] R.W. Motl, E. McAuley, Y. Suh Validity, invariance, and responsiveness of a self-report measure of functional limitations and disability in multiple sclerosis Disabil. Rehabil., 32 (2010), pp. 1260-1271

[39] R.W. Motl, E.M. Snook, E. McAuley, J.A. Scott, M.L. Douglass Correlates of physical activity among individuals with multiple sclerosis Ann. Behav. Med., 32 (2006), pp. 154-161

[40] E. McAuley, K.S. Courneya, D.L. Rudolph, C.L. Lox Enhancing exercise adherence in middle-aged males and females Prev. Med., 23 (1994), pp. 498-506 
[41] E. McAuley, R.W. Motl, K.S. Morris, L. Hu, S.E. Doerksen, S. Elavsky, J.F. Konopack Enhancing physical activity adherence and well-being in multiple sclerosis: a randomised controlled trial Mult. Scler., 13 (2007), pp. 652-659

[42] D.C. Mohr, P. Cuijpers, K. Lehman Supportive accountability: a model for providing human support to enhance adherence to eHealth interventions J. Med. Internet Res., 13 (2011), Article e30

[43] D.C. Mohr, B. Spring, K.E. Freedland, V. Beckner, P. Arean, S.D. Hollon, J. Ockene, R. Kaplan The selection and design of control conditions for randomized controlled trials of psychological interventions Psychother. Psychosom., 78 (2009), pp. 275-284

[44] T.E. Duncan, S.C. Duncan, L.A. Strycker, F. Li, A. Alpert An Introduction to Latent Variable Growth Curve Modeling: Concepts, Issues, and Applications Erlbaum, Hanwah, New Jersey (1999)

[45] C.E. Lance, R.J. Vandenberg, R.M. Self Latent growth models of individual change: the case of newcomer adjustment Organ. Behav. Hum. Decis. Process., 83 (2000), pp. 107-140

[46] J.B. Willett, A.G. Sayer Using covariance structure analysis to detect correlates and predictors of individual change over time Psychol. Bull., 116 (1992), pp. 363-381

[47] L.K. Muthén, B.O. Muthén Mplus. Los Angeles: Author (1998-2004)

[48]C.K. Enders, D.L. Bandalos The relative performance of full information maximum likelihood estimation for missing data in structural equation models Struct. Equ. Model., 8 (2001), pp. 430-457

[49] L. Hu, P.M. Bentler Cutoff criteria for fit indices in covariance structure analysis: conventional criteria versus new alternatives Struct. Equ. Model., 6 (1999), pp. 1-55

[50] Y. Suh, M. Weikert, D. Dlugonski, B. Sandroff, R.W. Motl Social cognitive correlates of physical activity: findings from a cross-sectional study of adults with relapsing-remitting multiple sclerosis J. Phys. Act. Health, 8 (2011), pp. 626-635

[51]Y. Suh, M. Weikert, D. Dlugonski, S. Balantrapu, R.W. Motl Social cognitive variables as correlates of physical activity in persons with multiple sclerosis: findings from a longitudinal, observational study Behav. Med., 37 (2011), pp. 87-94

[52] New survey finds technology plays a critical role in the lives of people with multiple sclerosis yet many are not using it to overcome disease-related challenges [news release], Natl. Mult. Scler. Soc. (October 26, 2007) http://www.microsoft.com/presspass/features/2007/oct07/10-26ms.mspx, Accessed 25th Jul 2013

[53] L. Wardell, S. Hum, A.M. Laizner, Y. Lapierre Multiple sclerosis patients' interest in and likelihood of using online health-care services Int. J. MS. Care, 11 (2009), pp. 79-89

[54] A.L. Dunn, R.E. Andersen, J.M. Jakicic Lifestyle physical activity interventions. History, short- and long-term effects, and recommendations Am. J. Prev. Med., 15 (1998), pp. 398-412 